\title{
On the circularly polarized optical emission from AE Aquarii
}

\author{
N. R. Ikhsanov ${ }^{1,2}$, S. Jordan ${ }^{3,4}$, and N. G. Beskrovnaya ${ }^{2,5}$ \\ 1 Max-Planck-Institut für Radioastronomie, Auf dem Hügel 69, 53121 Bonn, Germany \\ 2 Central Astronomical Observatory of the Russian Academy of Science at Pulkovo, Pulkovo 65-1, \\ 196140 Saint-Petersburg, Russia \\ 3 Institut für Theoretische Physik und Astrophysik, Universität Kiel, 24098 Kiel, Germany \\ 4 Institut für Astronomie und Astrophysik, Sand 1, 72076 Tübingen, Germany, \\ e-mail: jordan@astro.uni-tuebingen.de \\ ${ }^{5}$ Isaac Newton Institute of Chile, St. Petersburg Branch, Russia
}

Received 19 November 2001 / Accepted 17 January 2002

\begin{abstract}
The reported nightly mean value of the circular polarization of optical emission observed from the close binary system AE Aqr is $0.06 \% \pm 0.01 \%$. We discuss the possibility that the observed polarized radiation is emitted mainly by the white dwarf or its vicinity. We demonstrate that this hypothesis is rather unlikely since the contribution of the white dwarf to the optical radiation of the system is too small. This indicates that the polarimetric data on AE Aqr cannot be used for the evaluation of the surface magnetic field strength of the white dwarf in this system.
\end{abstract}

Key words. stars: binaries: close - stars: magnetic fields - stars: white dwarfs - polarization stars: individual: AE Aqr

\section{Introduction}

Measurements of circularly polarized emission from the close binary system AE Aqr have been reported by three independent groups of authors. Cropper (1986) estimated the circular polarization in the optical to be at the level of $0.05 \% \pm 0.01 \%$. Similar value (0.06\%) have been derived by Stockman et al. (1992). Beskrovnaya et al. (1996) reported a value for the nightly mean of the circular polarization in the $V$ and $V+R$ passbands to be $p_{\text {mean }}=0.06 \% \pm 0.01 \%$.

The possible origin of the circularly polarized emission from AE Aqr was first discussed by Bastian et al. (1988) in the framework of the oblique rotator model (Patterson 1979). Within this model (which was widely accepted before 1994), AE Aqr is considered as an intermediate polar in which the radiation of the white dwarf is powered by the accretion of plasma onto its surface with the rate of $\sim 10^{16} \mathrm{~g} \mathrm{~s}^{-1}$. Assuming the observed polarized emission to be generated in the shock at the base of the accretion column due to the cyclotron mechanism, Bastian et al. (1988) estimated the magnetic field strength of the white dwarf to be in excess of $10^{6} \mathrm{G}$.

This result, however, turned out to be one of the first serious arguments against the accretion-powered white dwarf model of AE Aqr: if the surface field strength of

Send offprint requests to: N. R. Ikhsanov,

e-mail: ikhsanov@mpifr-bonn.mpg.de the white dwarf in AE Aqr is indeed $B_{*} \gtrsim 1 \mathrm{MG}$, its magnetospheric radius,

$R_{\mathrm{m}} \simeq 7.7 \times 10^{9} \kappa_{0.5} \dot{M}_{16}^{-2 / 7} M_{0.8}^{-1 / 7} R_{8.8}^{12 / 7}\left(\frac{B_{*}}{10^{6} \mathrm{G}}\right)^{4 / 7} \mathrm{~cm}$

is larger than the corotation radius,

$R_{\text {cor }}=1.5 \times 10^{9} M_{0.8}^{1 / 3} P_{33}^{2 / 3} \mathrm{~cm}$

by more than a factor of five. Here $\dot{M}_{16}$ is the mass accretion rate expressed in units of $10^{16} \mathrm{~g} \mathrm{~s}^{-1}, \kappa_{0.5}=\kappa / 0.5$ is accounting for the geometry of the accretion flow $^{1}$, and $M_{0.8}, R_{8.8}$ and $P_{33}$ are the mass, radius, and the spin period of the white dwarf expressed in units of $0.8 M_{\odot}$, $10^{8.8} \mathrm{~cm}$ and $33 \mathrm{~s}$, respectively (for the system parameters see Table 1 in Ikhsanov 2000). Under these conditions, the white dwarf is in the centrifugal inhibition regime and a steady accretion process onto its surface does not occur.

The hypothesis that the accretion power is not responsible for the emission of the white dwarf in AE Aqr got serious grounds after 1994. It was recognized that the intensity of radiation emitted from the polar caps of the white

\footnotetext{
1 The value of this parameter lies within the interval $0.5 \lesssim$ $\kappa \lesssim 1$, where $\kappa=0.5$ corresponds to the disk geometry of the accretion flow and $\kappa=1-$ to the spherical geometry (see e.g. Ghosh \& Lamb 1979).
} 
dwarf does not correlate with the flaring in the system (Eracleous et al. 1994). Furthermore, the X-ray spectrum of AE Aqr is soft and essentially differs from the hard Xray spectra of intermediate polars (Clayton \& Osborne 1995). Finally, the discovery of the rapid spin down of the white dwarf (de Jager et al. 1994) and the conclusion that no developed Keplerian accretion disk exists in the system (Wynn et al. 1997; Welsh et al. 1998) have left no doubts that AE Aqr is not compatible with the oblique rotator model and that no intensive plasma accretion onto the surface of the white dwarf occurs (for discussion see Ikhsanov 2001). Therefore, the basic assumptions used by Bastian et al. (1988) for the interpretation of the circularly polarized emission of AE Aqr are unacceptable and the origin of the polarized radiation in this system remains an unresolved problem.

In this paper we are investigating the validity of the suggestion that the observed circularly polarized radiation originates from the white dwarf or its vicinity.

\section{Contribution of the white dwarf to the circularly polarized radiation}

It is presently well established that the optical radiation from AE Aqr comes from at least three different sources: the K3-K5 main sequence companion, the white dwarf, and the extended region which is associated with the stream of material lost by the normal companion and ejected from the system due to propeller action by the white dwarf. The latter source manifests itself in the Balmer continuum and broad single-peaked emission lines. This source is observed to be highly variable and is assumed to be responsible for the unusual flaring behaviour of the system in the optical/UV (Eracleous \& Horne 1996; Wynn et al. 1997; Ikhsanov 2000). In contrast, investigations of the normal companion (Welsh et al. 1995) and the white dwarf (Eracleous et al. 1994) give no evidence for either significant variations of their brightness or any correlation between the intensity of radiation coming from these components and the flaring in the system.

\subsection{The contribution of the white dwarf to the optical radiation of $A E A q r$}

The visual light of AE Aqr is dominated mainly by the red dwarf: up to $95 \%$ during quiescent state of the system (van Paradijs et al. 1989; Bruch 1991; Welsh et al. 1995). The contribution of the extended source to the optical radiation of AE Aqr has been estimated by Eracleous \& Horne (1996) to amount to about 3\%-4\% during quiescence and up to $40 \%$ during the flaring state of the system. Thus, the optical radiation from the white dwarf proves to be a rather small fraction of the total optical radiation detected from the system, namely, about 1\%-2\% during the quiescent state and smaller than $1 \%$ during the flaring state.

This conclusion is in a good agreement with the model of the white dwarf atmosphere reconstructed by Eracleous et al. (1994) from observations of AE Aqr with the Hubble telescope. Using the maximum entropy method, the authors constrained the average surface temperature of the white dwarf to the interval $10000 \mathrm{~K} \lesssim T_{\text {int }} \lesssim 16000 \mathrm{~K}$ and the temperature of the hot polar caps at the magnetic pole regions to $24000 \mathrm{~K} \lesssim T_{\max } \lesssim 28000 \mathrm{~K}$ (the projected area of the hot spots is $4.3 \times 10^{16} \mathrm{~cm}^{2}$; the distance to AE Aqr is adopted to be $100 \mathrm{pc}$ ). In the framework of this model, the contribution of the hot polar caps to the optical radiation of the system is about $0.1 \%-0.2 \%$ (that is just the observed amplitude of the $33 \mathrm{~s}$ coherent oscillations, Patterson 1979; de Jager et al. 1994) and the total contribution of the white dwarf is smaller than $2 \%$. This indicates that the optical radiation coming from the hot polar caps of the white dwarf is diluted by a factor of $k_{\text {cap }}=500-1000$ and the radiation of the white dwarf as a whole - by a factor of $k_{\mathrm{wd}}=50-100$.

\subsection{Pulse-averaged polarization}

It is important to note that the reported $p_{\text {mean }}$ is the average value of the circular polarization over a night. The errors of the individual measurements are too large to be of any significance (especially on the time scale of the white dwarf spin period, $P=33 \mathrm{~s}$ ). Moreover, the analysis of the polarimetric data obtained by Beskrovnaya et al. (1996) has shown no signs of any periodicity. In this situation the idea that the polarized radiation detected from AE Aqr comes mainly from the white dwarf or its vicinity should be considered as an assumption rather than a well justified assertion. If, nevertheless, we adopt this assumption, it is necessary to take into account that the reported degree of $0.06 \%$ is not only the nightly mean but also the pulse-averaged value. In this context, the polarization of radiation coming from an individual magnetic pole should be larger than the reported value $p_{\text {mean }}$.

As the white dwarf rotates, its northern and southern magnetic poles are successively seen. The contribution of each of the poles to the system radiation depends on the configuration of the white dwarf magnetic field, physical parameters of plasma in the pole regions, and the system geometry. Most of these parameters have been evaluated by Eracleous et al. (1994) from the analysis of the UV-spectrum and profiles of the $33 \mathrm{~s}$ coherent oscillations. According to their results, the longitudinal difference between the hot polar caps is $180^{\circ}$, strongly suggesting a dipolar geometry of the magnetic field of the white dwarf. No evidence for any significant differences in the physical parameters of the plasma situated at the northern and southern poles has been found. Finally, assuming that the rotational axis of the white dwarf is almost perpendicular to the orbital plane, they evaluated the angle between the magnetic and the rotation axes as $\beta \sim 75^{\circ}$ and the orbital inclination of the system to be $i \simeq 60^{\circ}$ (see also Patterson 1979).

Under these conditions, radiation coming from the northern and southern magnetic poles is circularly polarized to approximately the same degree but in opposite 


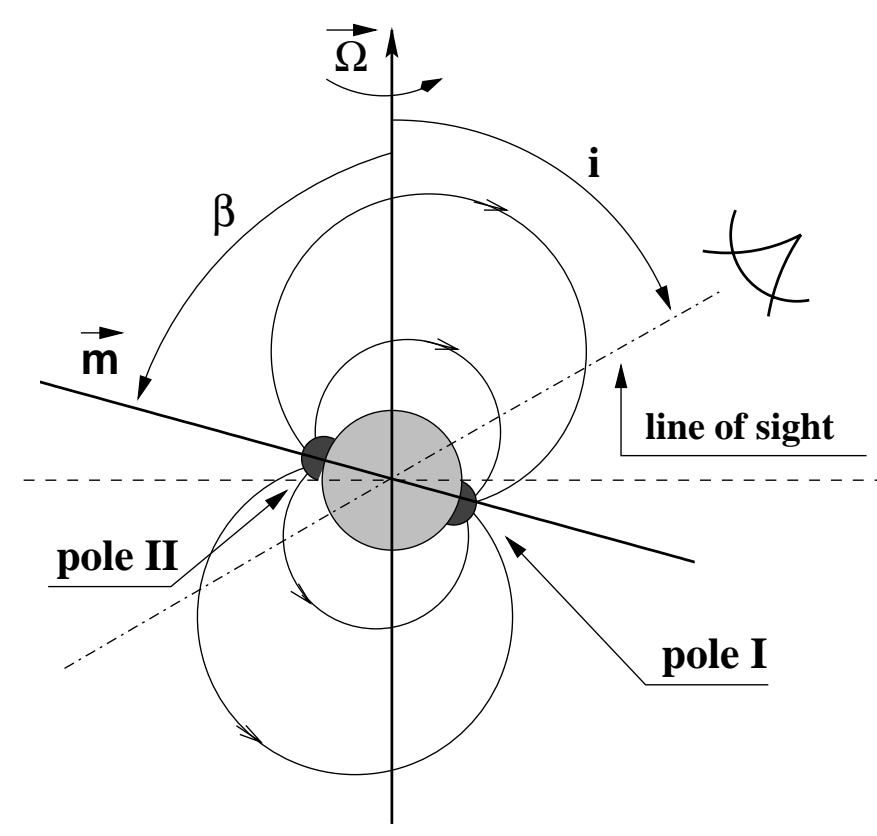

Fig. 1. The relative geometry of the rotational and magnetic axes of the white dwarf and the line of sight (see text).

directions: $p_{\mathrm{int}}^{\mathrm{n}} \simeq-p_{\mathrm{int}}^{\mathrm{s}}$. This indicates that the contributions of the northern and southern poles to the mean value of the circular polarization observed from the system have opposite signs and hence partly compensate each other. The value of the non-compensated (i.e. pulse-averaged) polarization depends on the system geometry.

The observed value of the polarization of an individual pole reaches its maximum when the angle between the magnetic axis and the line of sight is minimal and decreases to zero when this angle increases to $90^{\circ}$ (complete cancellation). In our case the minimum angle between the line of sight and the magnetic axis is $45^{\circ}$ for the pole I and is $15^{\circ}$ for the pole II (see Fig. 1). This indicates that the contribution of the pole II to the mean polarization detected from the system is larger than the contribution of the poleI. In the first approximation, the lower limit of the contribution of the pole I can be estimated by that part of the contribution of the pole II when the latter is observed within the following intervals of angles $\left[-90^{\circ},-45^{\circ}\right]$ and $\left[90^{\circ}, 45^{\circ}\right]$. Hence, the contribution of the pole II is not compensated only when the angle between the magnetic axis and the line of sight is $|\gamma| \lesssim 45^{\circ}$. In other words, the uncompensated circularly polarized emission is observable only during $1 / 4$ of the white dwarf spin period and hence the intrinsic polarization of radiation coming from an individual pole is at least by a factor of $k_{\mathrm{pa}}=4$ larger than the observed value.

\subsection{Intrinsic polarization}

From the arguments of two previous subsections one can discard the possibility that the hot polar caps, situated in the magnetic pole regions of the white dwarf, are the sources of the circularly polarized optical emission detected from AE Aqr: if the observed polarized radiation were generated at the polar caps, the intrinsic polarization of the source, $p_{\text {caps }}=k_{\text {caps }} \times k_{\text {pa }} \times p_{\text {mean }}$, should be in excess of $100 \%$ independent of the polarization mechanism!

There is, however, an alternative possibility. Namely, one can assume that the circularly polarized radiation is generated in the white dwarf atmosphere due to the linear and quadratic Zeeman effect. In this case the effective area of the source is significantly larger than the projected area of the hot polar caps. Therefore the intrinsic polarization can be limited to the interval $p_{\mathrm{wd}}=k_{\mathrm{wd}} \times k_{\mathrm{pa}} \times p_{\text {mean }} \gtrsim$ $12 \%$.

In order to test this possibility we estimated the expected value of the circularly polarized emission from a non-accreting white dwarf with the average surface temperature of $15000 \mathrm{~K}$, and two hot spots $(T \sim 25000 \mathrm{~K})$ with the effective projected area of $4.3 \times 10^{16} \mathrm{~cm}^{2}$, situated at the magnetic pole regions. The code developed by Jordan (1992; see also Putney \& Jordan 1995) has been used to compute the polarization degree at different wavelengths for an angle of $15^{\circ}$ between the magnetic axis and the line of sight, corresponding to the maximum value of the circular polarization. The results for two values of the surface magnetic field strength, $1 \mathrm{MG}$ and $50 \mathrm{MG}$, are plotted in Figs. 2 and 3, respectively. As can be seen from these figures, the expected maximum value of the polarization in the $V$ and $V+R$ passbands does not exceed $3 \%$, i.e. is at least by a factor of 4 smaller than required for the interpretation of the circular polarization observed from the system (the average polarization calculated within the interval $|\gamma|<45^{\circ}$ is smaller than the presented maximum value at least by a factor of 3 ).

\section{Discussion}

We have shown that the white dwarf cannot be considered as the main source of the circularly polarized optical emission detected from AE Aqr. The basic argument is a very small value of the white dwarf contribution to the visual light of the system. As a consequence, the circularly polarized radiation emitted by the white dwarf is significantly diluted and the expected value of the pulse-averaged circular polarization proves to be essentially smaller than the observed value.

Why was this argument not taken into account in previous investigations? The reason was the widely accepted but wrong assumption that the system radiation is powered by the accretion of material onto the white dwarf surface. Within this assumption, the contribution of the white dwarf to the system radiation proves to be overestimated by almost an order of magnitude and thus, the problem discussed in this paper simply does not arise. The incorrectness of this approach has been recognized only a few years ago, mainly due to the discovery of the rapid spin-down of the white dwarf (de Jager et al. 1994), detailed investigations of the optical/UV properties of the $33 \mathrm{~s}$ coherent oscillations (Eracleous et al. 1994) and the 


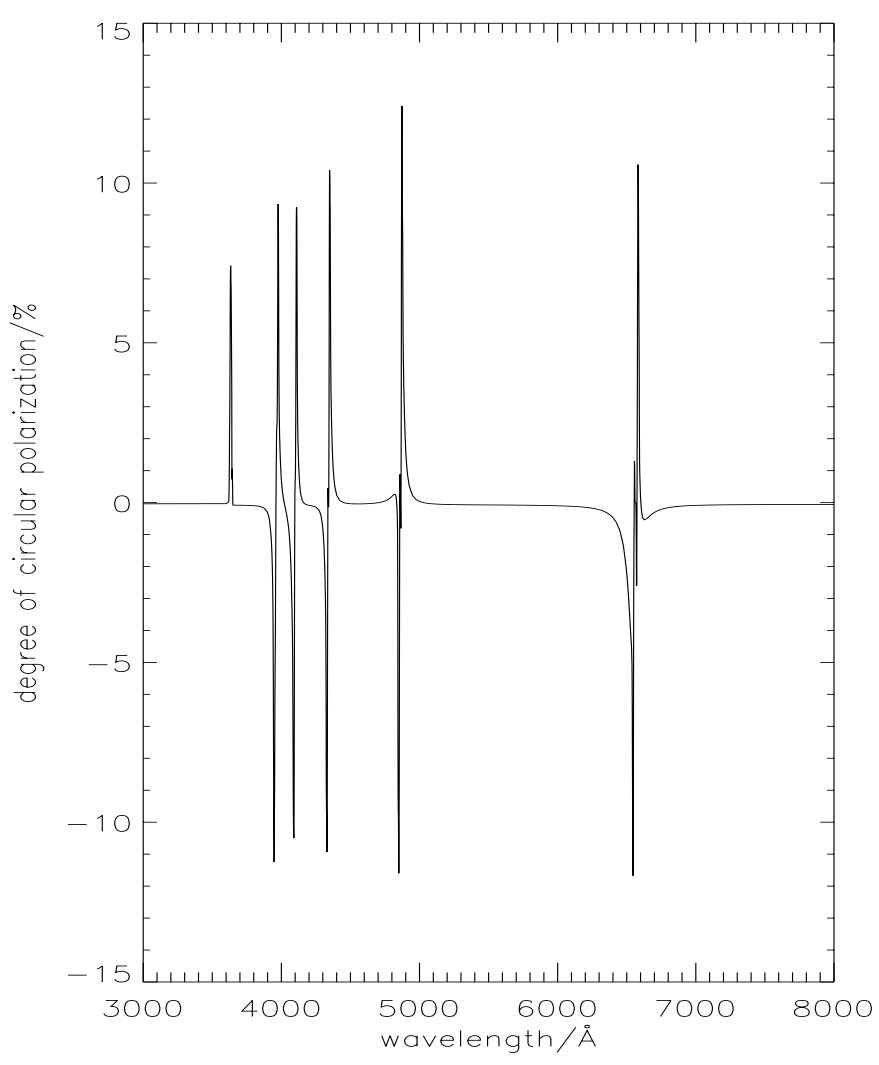

Fig. 2. The calculated circular maximum possible polarization of the white dwarf in AE Aqr due to Zeeman effect. The model parameters: $T_{\mathrm{int}}=15000 \mathrm{~K}, T_{\max }=25000 \mathrm{~K}, \gamma=15^{\circ}$ and $B_{*}=1 \mathrm{MG}$. Note that the polarization in AE Aqr is diluted at least by a factor of 200 compared to this prediction.

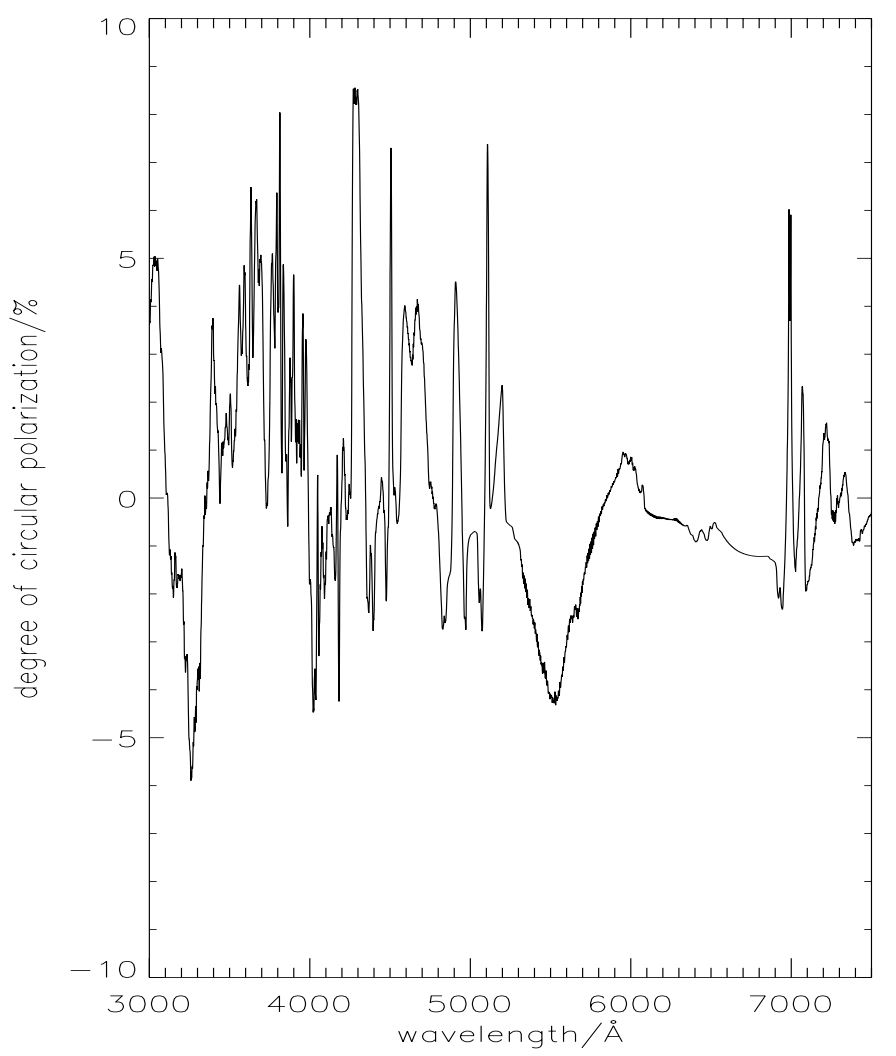

Fig. 3. The same as Fig. 2 for $B_{*}=50 \mathrm{MG}$. reconstruction of the diskless mass transfer picture (Wynn et al. 1997).

One of the important consequences of our conclusion is that the observed circularly polarized emission from AE Aqr cannot be used to estimate the surface magnetic field strength of the white dwarf. In the light of modern views on the system, neither the lower limit suggested by Bastian et al. (1988) nor the upper limit given by Stockman et al. (1992) to the magnetic field strength of the white dwarf can be used. In this situation, the value of the white dwarf magnetic field should be estimated using different methods (see e.g. Ikhsanov 1998).

Finally, we would like to note that except for the uncertain nightly mean value, no information about the properties of the circularly polarized emission from AE Aqr is currently available. In this situation, the justification of any alternative ideas about the origin of the polarized emission is very complicated and perhaps even impossible, until highly time resolved polarimetric observations with large signal-to-noise ratio are performed.

Acknowledgements. We would like to thank Boris Gaensicke for useful comments. NRI acknowledge the support of the Alexander von Humboldt Foundation within the Long-term Cooperation Program. Work on magnetic white dwarfs in Kiel was supported by the DFG under KO-738/7-1.

\section{References}

Bastian, T. S., Dulk, G. A., \& Chanmugam, G. 1988, ApJ, 324, 431

Beskrovnaya, N. G., Ikhsanov, N. R., Bruch, A., \& Shakhovskoy, N. M. 1996, A\&A, 307, 840

Bruch, A. 1991, A\&A, 251, 59

Clayton, K. L., \& Osborne, J. P. 1995, in Magnetic Cataclysmic Variables, ed. D. Buckley, \& B. Warner, ASP Conf. Ser., 85,379

Cropper, M. 1986, MNRAS, 222, 225

de Jager, O. C., Meintjes, P. J., O'Donoghue, D., \& Robinson, E. L. 1994, MNRAS, 267, 577

Eracleous, M., \& Horne, K. 1996, ApJ, 471, 427

Eracleous, M., Horne, K., Robinson, E. L., et al. 1994, ApJ, 433,313

Ghosh, P., \& Lamb, F. K. 1979, ApJ, 232, 259

Ikhsanov, N. R. 1998, A\&A, 338, 521

Ikhsanov, N. R. 2000, A\&A, 358, 201

Ikhsanov, N. R. 2001, A\&A, 374, 1030

Jordan, S. 1992, A\&A, 265, 570

Patterson, J. 1979, ApJ, 234, 978

Putney, A., \& Jordan, S. 1995, ApJ, 449, 863

Stockman, H. S., Schmidt, G. D., Berriman, G., et al. 1992, ApJ, 401, 628

van Paradijs, J., Kraakman, H., \& van Amerongen, S. 1989, A\&AS, 79, 205

Welsh, W. F., Horne, K., \& Gomer, R. 1995, MNRAS, 275, 649

Welsh, W. F., Horne, K., \& Gomer, R. 1998, MNRAS, 298, 285

Wynn, G. A., King, A. R., \& Horne, K. 1997, MNRAS, 286, 436 